\title{
Stimulated resonant $x$-ray Raman scattering with incoherent radiation
}

\author{
Clemens Weninger ${ }^{*}$ and Nina Rohringer ${ }^{\dagger}$ \\ Max Planck Institute for the Physics of Complex Systems, 01187 Dresden, Germany \\ and Center for Free Electron Laser Science, 22607 Hamburg, Germany
}

(Received 10 October 2013; published 21 November 2013)

\begin{abstract}
We present a theoretical study on stimulated electronic Raman scattering in neon by resonant excitation with an $\mathrm{X}$-ray free electron laser (XFEL). This study is in support of the recent experimental demonstration [C. Weninger et al., Phys. Rev. Lett. (to be published)] of stimulated x-ray Raman scattering. Focusing the broadband XFEL pulses into a cell of neon gas at atmospheric pressure a strong inelastic $\mathrm{X}$-ray scattering signal in the forward direction was observed, as the x-ray energy was varied across the region of core-excited Rydberg states and the $K$ edge. The broadband and intrinsically incoherent x-ray pulses from the XFEL lead to a rich, structured line shape of the scattered radiation. We present a generalized Maxwell-Liouville-von Neumann approach to self-consistently solve for the amplification of the scattered radiation along with the time evolution of the density matrix of the atomic and residual ionic system. An in-depth analysis of the evolution of the emission spectra as a function of the Raman gain is presented. Furthermore, we propose the use of statistical methods to obtain high-resolution scattering data beyond the lifetime broadening despite pumping with incoherent $\mathrm{x}$-ray pulses.
\end{abstract}

DOI: 10.1103/PhysRevA.88.053421

PACS number(s): $33.35 .+r, 33.20 . X x$

Optical nonlinear Raman spectroscopy is a powerful tool to study dynamical processes and excitations in atoms and molecules [1,2]. With the advance of X-ray free electron lasers (XFEL) [3-7] it is possible to produce powerful x-ray pulses with femtosecond durations which in principle would allow the transfer of Raman spectroscopy into the $\mathrm{x}$-ray region [8-11]. In resonant x-ray Raman scattering, also referred to as resonant inelastic x-ray scattering (RIXS), core electrons are resonantly excited to valence orbitals with X-ray pulses. The decay of the short-lived, core-excited state can proceed either by an Auger decay or by a radiative decay, thereby emitting a photon with red-shifted frequency [12]. In the soft $\mathrm{x}$-ray region, the Auger decay typically dominates and $\mathrm{x}$-ray scattering cross sections are comparatively small. Stimulating the x-ray scattering process by providing "pump" and "dump" photons the scattering signal can be greatly amplified. Cross sections for stimulated $\mathrm{x}$-ray scattering have been theoretically estimated [13-15], but the lack of intense x-ray sources have so far precluded demonstration. In a recent experiment stimulated X-ray Raman scattering was experimentally observed [11] with an XFEL. Here, we present a detailed theoretical description, underlying the interpretation of the experimental data.

Ideally, a stimulated x-ray Raman experiment would require a two-color x-ray source of well-defined photon energies and narrow spectral bandwidth to specifically target electronic transitions in the resonant scattering process. Alternatively, stimulated x-ray Raman scattering could be observed by an ultrashort, transform limited x-ray pulse with a bandwidth covering the inelastic energy transfer. In the latter scheme, referred to as impulsive Raman scattering, typically more than one electronic excitation and decay channel will be probed and electronic wave packets are created. The inelastic scattering process is observed by small, reproducible changes in the spectrum of the transmitted radiation. Unfortunately, at the moment

\footnotetext{
*clemens.weninger@pks.mpg.de

†nina@pks.mpg.de
}

their are no such $\mathrm{x}$-ray sources available which deliver either narrowband two-color X-ray pulses or reproducible, transformlimited attosecond pulses to perform such an experiment. Current XFELs are based on the self-amplified spontaneous emission (SASE) principle [16], starting from shot noise in the electron bunches. SASE pulses are characterized by a relatively broad bandwidth (typically $0.1 \%$ to $1 \%$ of the central photon energy) of limited longitudinal coherence. This results in emission spectra which vary on a shot-to-shot basis [17] and are very spiky in both time and frequency domain $[18,19]$ (see Figs. 3(b) and 3(d) as an example for the temporal and spectral intensity envelope of a typical SASE pulse). The recent experimental demonstration of Raman spectroscopy with these SASE pulses proved very challenging [11], since the broadband XFEL pulses, overlapping with both the region of core-excited resonances and the continuum above the $K$ edge, made it difficult to disentangle stimulated resonant scattering from stimulated amplification of fluorescence following photoionization. Differentiating between both effects required a detailed analysis of the emission line shapes and the comparison of the experimentally detected signal levels to a quantitative theory of stimulated x-ray scattering with incoherent XFEL radiation. Here, we give an in-depth presentation of the theory which underlies the interpretation of the experimental results in Ref. [11]. We present a MaxwellLiouville-von Neumann approach to predict the emission spectra of stimulated $\mathrm{x}$-ray Raman scattering in a dense gas of atomic neon. Moreover, we propose a method to obtain high-resolution scattering data using broadband stochastic pulses in combination with statistical analysis.

\section{SUMMARY OF EXPERIMENTAL RESULTS}

In the experiment [11], XFEL pulses of $\approx 40 \mathrm{fs}$ in duration were focused into a 10 -mm-long gas cell filled with neon at a pressure of approximately 500 Torr (corresponding to an atomic density of $\approx 1.6 \times 10^{19} \mathrm{~cm}^{-3}$ ). The focal region is approximately 1-2 $\mu \mathrm{m}$ wide and $15 \mathrm{~mm}$ long, so that upon irradition with the XFEL beam a long, narrow plasma channel 


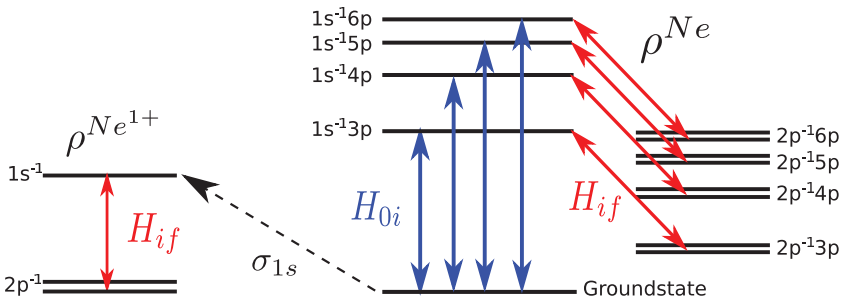

FIG. 1. (Color online) Level scheme of neon. The blue arrows $H_{0 i}$ show the resonant coupling of the XFEL pump pulse to the core-excited intermediate state. The black dotted lines represent photoionization of core electrons yielding singly charged neon $\mathrm{Ne}^{1+}$. The red arrows $H_{i f}$ represent the coupling of the core-excited intermediate state to the final states. This leads to stimulated fluorescence in the case of $\mathrm{Ne}^{1+}$ and stimulated scattering for the neutral neon.

of core-excited neon atoms is created. The neon atoms are core excited in a longitudinal pump geometry, as the XFEL pulse propagates through the gas sample. In the experiment, the XFEL photon energy was varied across the $K$ edge $(870.2 \mathrm{eV})$ of neon and the emitted $\mathrm{x}$-ray radiation was detected in the forward direction with an x-ray spectrometer. A level scheme of neon can be seen in Fig. 1 . The strongest pre- $K$-edge resonance of neon, corresponding to the $1 s-3 p$ transition, is roughly at $867 \mathrm{eV}$, i.e., only $3 \mathrm{eV}$ below the K-edge at $870.2 \mathrm{eV}$ [20]. The core-excited resonances have a width of only $0.27 \mathrm{eV}$, determined by the Auger decay, which typically happens on a time scale of $2.4 \mathrm{fs}$. In the experiment, the SASE bandwidth was measured to be $7 \mathrm{eV}$. Tuning the incoming XFEL beam to the $1 s-3 p$ resonance, hence, resulted in a spectral overlap with the whole Rydberg series of core-excited states ( $1 s$ core electrons excited to valence orbitals $3 p, 4 p, 5 p$, etc.) as well as with the continuum. Coupling to the continuum results in photoionization of the neon atoms and drives amplification of the $K \alpha$ fluorescence $[21,22]$. Coupling to the resonance region results in stimulated inelastic $x$-ray scattering, where the spectral tail of the XFEL pulse provides seed photons on the emission energy at around $850 \mathrm{eV}$. A detailed analysis of the experimental emission line shapes revealed a reproducible line shape and line position for photon energies $>870 \mathrm{eV}$, whereas for $<870 \mathrm{eV}$, i.e., in the region of the resonances, a stochastic shift, broadening and sometimes multipeak structure of the emission line was observed. As we show in this study, the stochastic line shift and multipeak structure is a signature of resonant Raman scattering with incoherent $\mathrm{x}$-ray radiation.

\section{THEORETICAL APPROACH}

To simulate the scattering process a one-dimensional Maxwell-Liouville-von Neumann approach was developed, a generalization of Maxwell-Bloch models to study laser amplification [23-25]. Due to the elongated geometry we assume the paraxial wave approximation. The transverse coordinates are therefore neglected and only the direction of the propagation along the $z$ axis is considered. The neon atoms are supposed to be initially in the ground state. The XFEL pulse can either ionize core electrons leading to $\mathrm{Ne}^{1+}$ or resonantly excite core electrons to the unoccupied orbitals. To treat both cases on an equal footing we introduce two density matrices: a truncated density matrix $\rho$ for atomic neon and a reduced ionic density matrix $\rho^{1+}$ describing the residual singly charged neon ion. The atomic neutral density matrix comprises coreexcited states $1 s^{-1} n p^{1}{ }^{1} P_{1}$ to the $3 p$ up to the $6 p$ level and final scattering state doublets $2 p^{-1} n p^{1}{ }^{1} D_{2}$ and $2 p^{-1} n p^{1}{ }^{1} S_{0}$ with a hole in the $2 p$ shell and a spectator electron in the levels $3 p$ to $6 p$. The states covered by the ionic density matrix $\rho^{1+}$ are the core-excited ionic state $1 s^{-1}{ }^{2} S_{1 / 2}$ and the valence excited state doublet $2 p^{-12} P_{1 / 2}, 2 p^{-1}{ }^{2} P_{3 / 2}$. Table I shows all relevant transitions along with their energies and dipole moments.

In our model we solve for the time evolution of the two density matrices coupled to a classical electric field. Since the XFEL is linearly polarized and seeds the amplification process we assume the XFEL and the scattered radiation are both linearly polarized in the same direction. The equations are solved in a moving window [27] by transforming to the retarded time $\tau=t-\frac{z}{c}$, making the calculations more efficient. To treat absorption losses of the XFEL radiation resulting from both resonant excitation and ionization we need to account for the steplike behavior of the $1 s$ photoionization cross section around the $K$ edge. Since we adopt for a real-time propagation approach to study the propagation and inelastic scattering process, unlike a formulation in the frequency domain by response functions, the spectrum of the electric field is not accessible during the simulation. In the time domain, however, a steplike absorption coefficient at the $K$ edge is difficult to introduce. Hence we split the initial SASE spectrum in two parts, a part below the $K$ edge and a part above the $K$ edge, which will be treated separately in the propagation scheme. The part above the $K$ edge $J_{p}(\tau, z)$ is solely described by the evolving flux

$$
\frac{\partial J_{p}(\tau, z)}{\partial z}=-\rho_{00}(\tau, z) n \sigma_{\mathrm{tot}} J_{p}(\tau, z),
$$

which gets attenuated by photoabsorption. Here, $n$ denotes the density of the neon gas, $\rho_{0,0}(\tau, z)$ is the time-dependent population of neon in the ground state, and $\sigma_{\text {tot }}$ is the total photoionization cross section, including ionization of the core and valence shells. The part below the $K$ edge and the scattered radiation are treated by a single classical electric field,

$$
E(t, z)=\mathscr{E}(t, z) e^{-i \omega t}+\mathscr{E}^{\star}(t, z) e^{i \omega t},
$$

which is split into a slowly varying envelope $\mathscr{E}$ and a fast oscillating exponent. In contrast to treatments of the spontaneous Raman effect, which is often treated by a quantum field [28], we treat the field classically. Since the Raman process is seeded by the tails of the XFEL pulse, which typically has intensities overcoming the vacuum fluctuations of the electromagnetic field, a classical description should be valid. Spontaneous Raman scattering is hence neglected in our approach. Using the second-order wave equation for the electric field and assuming the slowly varying envelope approximation [29] the envelope evolves according to

$$
\frac{\partial \mathscr{E}(\tau, z)}{\partial z}=i \frac{2 \pi \omega}{c} \mathscr{P}(\tau, z),
$$

where $\mathscr{P}$ is the macroscopic polarization of the neon gas. Equation (3) is written with respect to the frame of the propagating pulse, and $\tau=t-z / c$ denotes the retarded time. 
TABLE I. Transition energies and dipole moments, calculated with the Cowan code [26].

\begin{tabular}{|c|c|c|c|}
\hline Excitation & Transition & Energy (eV) & Transition dipole (a.u.) \\
\hline \multirow[t]{5}{*}{$\mathrm{Ne}$} & $|0\rangle \rightarrow 1 s^{-1} 3 p^{1} P_{1}$ & 867.5 & $7.7 \times 10^{-3}$ \\
\hline & $|0\rangle \rightarrow 1 s^{-1} 4 p^{1} P_{1}$ & 869.2 & $4.4 \times 10^{-3}$ \\
\hline & $|0\rangle \rightarrow 1 s^{-1} 5 p^{1} P_{1}$ & 869.8 & $3.0 \times 10^{-3}$ \\
\hline & $|0\rangle \rightarrow 1 s^{-1} 6 p^{1} P_{1}$ & 870.0 & $2.2 \times 10^{-3}$ \\
\hline & $K$ edge & 870.2 & \\
\hline \multicolumn{4}{|l|}{ Emission } \\
\hline \multirow[t]{8}{*}{$\mathrm{Ne}$} & $1 s^{-1} 3 p^{1} P_{1} \rightarrow 2 p^{-1} 3 p^{1} S_{0}$ & 849.3 & $4.8 \times 10^{-2}$ \\
\hline & $1 s^{-1} 3 p^{1} P_{1} \rightarrow 2 p^{-1} 3 p^{1} D_{2}$ & 849.7 & $4.0 \times 10^{-2}$ \\
\hline & $1 s^{-1} 4 p^{1} P_{1} \rightarrow 2 p^{-1} 4 p^{1} S_{0}$ & 849.6 & $4.5 \times 10^{-2}$ \\
\hline & $1 s^{-1} 4 p^{1} P_{1} \rightarrow 2 p^{-1} 4 p^{1} D_{2}$ & 849.7 & $4.2 \times 10^{-2}$ \\
\hline & $1 s^{-1} 5 p^{1} P_{1} \rightarrow 2 p^{-1} 5 p^{1} S_{0}$ & 849.7 & $3.8 \times 10^{-2}$ \\
\hline & $1 s^{-1} 5 p^{1} P_{1} \rightarrow 2 p^{-1} 5 p^{1} D_{2}$ & 849.7 & $4.1 \times 10^{-2}$ \\
\hline & $1 s^{-1} 6 p^{1} P_{1} \rightarrow 2 p^{-1} 6 p^{1} S_{0}$ & 849.7 & $3.3 \times 10^{-2}$ \\
\hline & $1 s^{-1} 6 p^{1} P_{1} \rightarrow 2 p^{-1} 6 p^{1} D_{2}$ & 849.7 & $4.0 \times 10^{-2}$ \\
\hline \multirow[t]{2}{*}{$\mathrm{Ne}^{1+}$} & $1 s^{-12} S_{1 / 2} \rightarrow 2 p^{-12} P_{1 / 2}$ & 849.8 & $4.8 \times 10^{-2}$ \\
\hline & $1 s^{-12} S_{1 / 2} \rightarrow 2 p^{-12} P_{3 / 2}$ & 849.8 & $6.8 \times 10^{-2}$ \\
\hline
\end{tabular}

The polarization is proportional to the sum of the off-diagonal density matrix elements,

$$
\mathscr{P}=2 n\left(\sum_{i \neq j} z_{i j} \rho_{i j}^{1+} e^{i\left(\omega-\omega_{i j}\right) t}+\sum_{i \neq j} z_{i j} \rho_{i j} e^{i\left(\omega-\omega_{i j}\right) t}\right),
$$

where $z_{i j}$ is the corresponding dipole matrix element for that transition, here $i$ and $j$ denote a composite index identifying the states of the atomic and ionic system.

The master equations for the time evolution of the density matrices can be derived by an open quantum system approach. Details of the approach can be found in Ref. [30] and we adapt it to account for the case of two density matrices for the neutral neon and the ion. We give a short summary of the derivation here: Starting from the configuration interaction approach we expand the wave function in the interaction picture $|\Psi, t\rangle_{I}$ in terms of the ground state $\left|\phi_{0}\right\rangle$ and states $\left|\phi_{i}^{a} J\right\rangle$, which are constructed from the ground state by exciting an initially occupied single orbital $i$ into an initially unoccupied orbital $a$, forming a state of total angular momentum $J$ :

$$
|\Psi, t\rangle_{I}=\alpha_{0}(t)\left|\phi_{0}\right\rangle+\sum_{i, a, J} \alpha_{i}^{a}(t)\left|\phi_{i}^{a} J\right\rangle .
$$

The matter-field interaction potential is given in the dipole approximation by $H_{I}=-\mu E(t)$. The time evolution of the expansion coefficients $\alpha_{0}(t)$ and $\alpha_{i}^{a} J(t)$ is determined by the time-dependent Schrödinger equation in the interaction picture and is given by

$$
\begin{aligned}
\dot{\alpha}_{0}= & i \sum_{j, a, J} z_{0,{ }_{j}^{a} J} \alpha_{j}^{a} J(t) E(t) e^{-i \omega_{a j} t} \\
\dot{\alpha}_{i}^{a} J= & i z_{i}^{a} J, 0 \\
& \alpha_{0}(t) E(t) e^{i \omega_{a i} t}+i \sum_{k} z_{i}^{a} J,_{k}^{a} J \alpha_{k}^{a} J(t) E(t) e^{i\left(\omega_{a i}-\omega_{a k}\right) t} \\
& -\frac{\Gamma_{i}}{2} \alpha_{i}^{a} J(t) .
\end{aligned}
$$

Here, we phenomenologically introduced the Auger decay. Core-excited levels $i$ are decaying with a rate of $\Gamma_{i}=0.27 \mathrm{eV}$; $\Gamma_{i}$ is zero for all other states. In a next step, we differentiate between resonantly core-excited states into orbital $a$ and orbitals $a$ pertaining to the continuum. We define a reduced ionic density matrix $\rho^{1+}$ by a product of wave-function coefficients summed over continuum states $a$ :

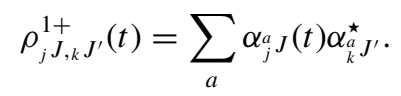

The remainder of the single-particle excitations and the ground state are included in the atomic density matrix of the neutral system:

$$
\rho_{j}^{a} J_{,}^{a_{k}^{\prime} J^{\prime}}(t)=\alpha_{j}^{a} J(t) \alpha_{a_{k}^{\prime} J^{\prime}}^{\star}
$$

Starting from these expressions, master equations for the ionic and atomic density matrices can be derived, following steps outlined in Ref. [30]. We get the following equations for the evolution of the diagonal elements (populations) of the atomic density matrix:

$$
\begin{aligned}
& \dot{\rho}_{0,0}=-\left[\sigma_{1 s} J_{p}+\sigma_{v}\left(J+J_{p}\right)\right] \rho_{00}-2 \operatorname{Im} \sum_{n} R_{0,1 s}^{n p} J \rho_{1 s}^{n p} J, 0, \\
& \dot{\rho}_{1 s}^{n p} J_{1 s}^{n p} J=-\Gamma \rho_{1 s}^{n p} J_{1, s}^{n p} J-2 \operatorname{Im}\left(R_{1 s}^{n p} J, 0 \rho_{0,1 s}^{n p} J+\sum_{J^{\prime}} R_{1 s}^{n p} J_{, 2 p}^{n p} J^{\prime} \rho_{1 s}^{n p} J^{\prime,}{ }_{1 s}^{n p} J\right), \\
& \dot{\rho}_{2 p}^{n p} J,{ }_{2 p}^{n p} J=-2 \operatorname{Im} R_{2 p}^{n p} J,{ }_{1 s}^{n p} K \rho_{1 s}^{n p} K,{ }_{2 p}^{n p} J .
\end{aligned}
$$


The coherences (off-diagonal matrix elements) in the neutral atom evolve according to

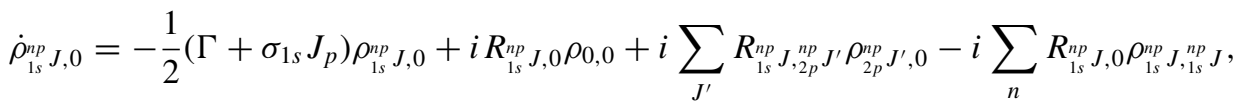

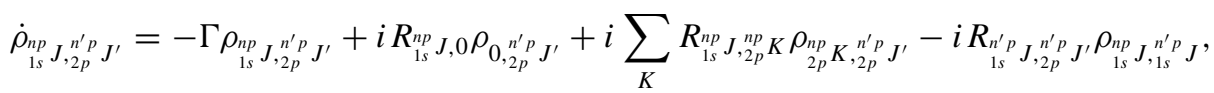

$$
\begin{aligned}
& \dot{\rho}_{\substack{n p \\
1 s, 1 s}}^{n^{\prime} p}=-\Gamma \rho_{1 s}^{n p} \rho_{1 s}^{n^{\prime} p} J \\
& \dot{\rho}_{n p} J_{2 p}^{n_{2 p}^{\prime} J^{\prime}}=i R_{2 p}^{n p} J_{1 s}^{n p} K \underset{1 s}{n \rho_{n p}} \rho_{2 p}^{n_{2}^{\prime} p} J^{\prime}-i R_{n^{\prime} p} K{ }_{2 p}^{n^{\prime} p} J^{\prime}{\underset{2 p}{n p} J_{1 s}^{n^{\prime} p} K}^{n_{1 s}}
\end{aligned}
$$

The population and coherences of the singly charged ion evolve according to

$$
\begin{aligned}
& \dot{\rho}_{i i}^{1+}=\sigma_{1 s} J_{p} \rho_{00}-\Gamma \rho_{i i}^{1+}-2 \operatorname{Im} \sum_{f} R_{i f} \rho_{f i}^{1+}, \\
& \dot{\rho}_{f f}^{1+}=\sigma_{2 p}\left[J+J_{p}\right] \rho_{00}+2 \operatorname{Im} R_{i f} \rho_{f i}^{1+}, \\
& \dot{\rho}_{i f}^{1+}=-\frac{\Gamma}{2} \rho_{i f}^{1+}+i \sum_{f^{\prime}} R_{i f^{\prime}} \rho_{f^{\prime} f}^{1+}-i R_{i f} \rho_{i i}^{1+},
\end{aligned}
$$

where $J=\frac{c}{8 \pi \omega}|\mathscr{E}|^{2}$ is the x-ray flux below the $K$ edge and

$$
R_{i j}=\frac{1}{2} z_{i j} \mathscr{E} \mathrm{e}^{-i\left(\omega-\left|\omega_{i j}\right|\right) t}=R_{j i}^{\star}
$$

is the complex Rabi frequency in the rotating wave and dipole approximation. $\omega$ is the frequency of the slowly varying envelope approximation and $\sigma_{k}$ is the ionization cross section for the corresponding state.

The density matrix equations are solved by a split-step method [31]. In this method the unitary evolution due to the Hamiltonian and the loss terms are solved separately. The parts involving the field interaction are solved by means of the discrete time evolution operator $U=e^{-i H_{I} \Delta t}$. The matrix exponential is calculated by diagonalization of the interaction Hamiltonian at each time step to ensure that $U$ is unitary. The Auger decay terms lead to an exponential decay and together with the photoionization terms are combined into the loss operator $\mathscr{D}$. We adopt a second-order splitting scheme where the density matrix is advanced by doing a half time step of the loss operator $\mathscr{D}$ followed by a full time step of the discrete time evolution operator and followed by another half time step of the loss operator. The density matrix equations are solved together with the Maxwell equation [Eq. (3)] and the pump flux equation [Eq. (1)] to obtain a self-consistent scheme.

To a good approximation the XFEL SASE pulses can be modeled as chaotic pulses with Gaussian statistics [18,32]. These pulses show a spiky pulse structure in the time and in the frequency domain as shown for a single SASE shot in Figs. 3(b) and 3(d). The number of modes (spikes) is conserved by transforming from the spectral to the temporal domain. The ensemble average of the pulses is characterized by the average spectral bandwidth $\Delta \omega$ and the average pulse duration $T$. The temporal coherence (average width of a spike in the time domain) is related to the total spectral width $T_{\text {coh }}=\frac{\sqrt{\pi}}{\Delta \omega}$ and the spectral coherence (average width of a spike in the frequency domain) is inversely proportional to the average pulse duration $\omega_{\text {coh }}=\frac{1}{T}[18,19]$. Throughout the paper we assume a Gaussian power spectrum for the ensemble average with a bandwidth of $\Delta \omega=7 \mathrm{eV}$ (FWHM). The pulses are generated by producing Gaussian random numbers with variances according to the power spectrum. The pulses are then Fourier transformed into time and multiplied by a Gaussian temporal envelope for the intensity $[33,34]$. We typically suppose a pulse duration of $40 \mathrm{fs}$ FHWM of the intensity.

\section{DISCUSSION OF RESULTS}

We now use this framework to study stimulated resonant Raman scattering. Figure 2 shows the amplification of the Raman scattered radiation with propagation through the medium for two ensembles of SASE pulses with different central photon energies. Shown are the number of photons in the down-scattered x-ray field (determined by integration of the spectral intensity from 847 to $851 \mathrm{eV}$ ) and the number of photons for photon energies $<870.2 \mathrm{eV}$ as a function of propagation distance. For both ensembles, we assumed a total of $1.5 \times 10^{12}$ photons in the incoming XFEL pulse and a flat focus with a radius of $2 \mu \mathrm{m}$. The part of the XFEL pump pulse below the $K$ edge gets only slightly attenuated with propagation. The narrow excitation resonances together with the small dipole moments result in a high transmission of around 0.5 for the considered central photon energies of 866 and $867 \mathrm{eV}$. The spectral tail of the XFEL provides $3 \times 10^{5}$

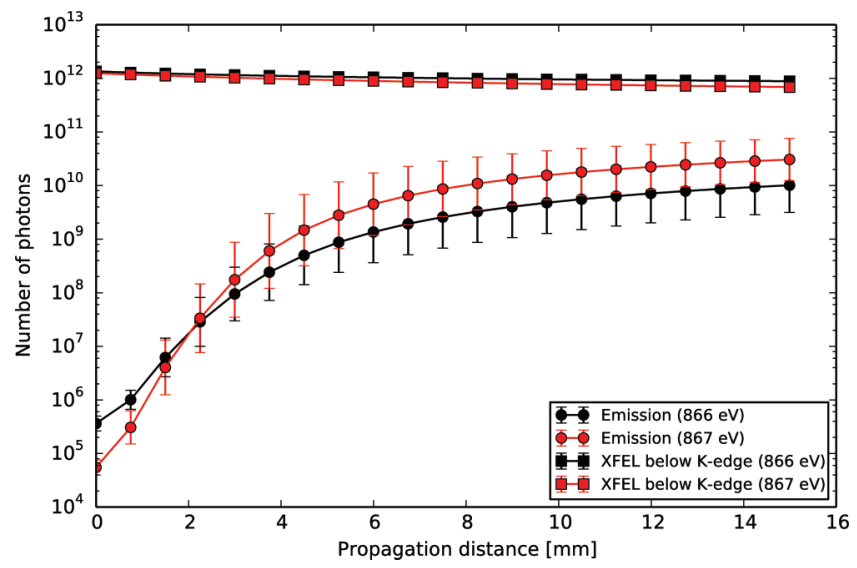

FIG. 2. (Color online) Number of photons in the emitted pulse and XFEL pulse as a function of propagation distance for two SASE ensembles with different central photon energies $866 \mathrm{eV}$ (black lines) and $867 \mathrm{eV}$ (red lines). The error bars represent the standard deviations of the ensemble. 
seed photons for the 866-eV pump pulse and $5 \times 10^{4}$ seed photons for $867 \mathrm{eV}$ in a spectral window of $4 \mathrm{eV}$ around the emission transition at the beginning of the medium $(z=0)$. The vacuum fluctuations of the quantum field would result in approximately 100 spontaneously Raman scattered photons in the entire interaction volume. The seed photons in the XFEL tail therefore greatly dominate over the vacuum fluctuations of the field. The pulse with $866-\mathrm{eV}$ central photon energy provides more seed photons than the pulse with $867 \mathrm{eV}$ since its central photon energy is closer to the emission transition of $\approx 849 \mathrm{eV}$. The gain for the pulse with $867-\mathrm{eV}$ central photon energy is, however, higher, because the peak of the spectral intensity is closer to the excitation resonances ranging from 867 up to the $K$ edge. The signal in the Raman scattered radiation shows a lethargic kick-off of the gain for low $z$. After going through an exponential gain region the signal bends over and the gain is reduced. This bending over of the gain curves does not necessarily imply saturation of the lasing transition, which is defined by an inelastic scattering rate which beats the Auger decay. Whereas the red curve for $867 \mathrm{eV}$ reaches true saturation towards the end of the medium, the black curve for $866 \mathrm{eV}$ levels off at a lower photon number, due to a depletion of the pump photons. The Raman gain stops, since the pump rate to the core-excited state drops. In the regime of high laser saturation, nonlinear effects change the emission lineshape and we even observe degenerate four-wave mixing. This four-wave mixing was also recently theoretically predicted in amplified $\mathrm{x}$-ray emission in molecules [35].

To study the scattering line shape and its evolution with propagation we focus in the following on the pulse with 866-eV central photon energy, which does not show saturation effects due to the lower gain. X-ray Raman scattering with a narrowband source (less than the lifetime width of the core-excited state) shows a linear dispersion between the incoming $\omega_{\text {in }}$ and outgoing radiation $[11,12,36]$. The energy of the outgoing radiation is

$$
\omega_{\mathrm{out}}=\omega_{\mathrm{in}}-\omega_{f 0}
$$

where $\omega_{f 0}$ is the energy difference between the final state and the ground state. In other words, the resonance scattering process of an incoming pulse detuned by $\Omega=\omega_{\text {in }}-\omega_{i 0}$ with respect to the excitation resonance $\omega_{i 0}$ results in a detuning of $\Omega$ from the resonance energy $\omega_{i f}$, an effect which was termed "anomalous" linear dispersion of resonance scattering. Hence, the outgoing radiation of frequency $\omega_{\text {out }}$ follows the detuning of the incoming field $\omega_{\text {in. }}$. The situation of resonance scattering with a spiky, broadband SASE spectrum is more complex. It is however possible to see the entire SASE spectrum as a sum of randomly phased narrowband, coherent spectral contributions. Each spectral spike has a specific detuning from resonance and the scattering process follows the anomalous linear dispersion. For a single SASE pulse and several resonances, this results in a multiple-spiked emission pattern. Since the spectral distribution of the SASE pulses changes from pulse to pulse, every representative of the SASE ensemble will have different detunings from resonance and hence a slightly different emission spectrum. This scenario can be visualized by looking at the evolution of the spectrum as a function of propagation length. The spectrum of the
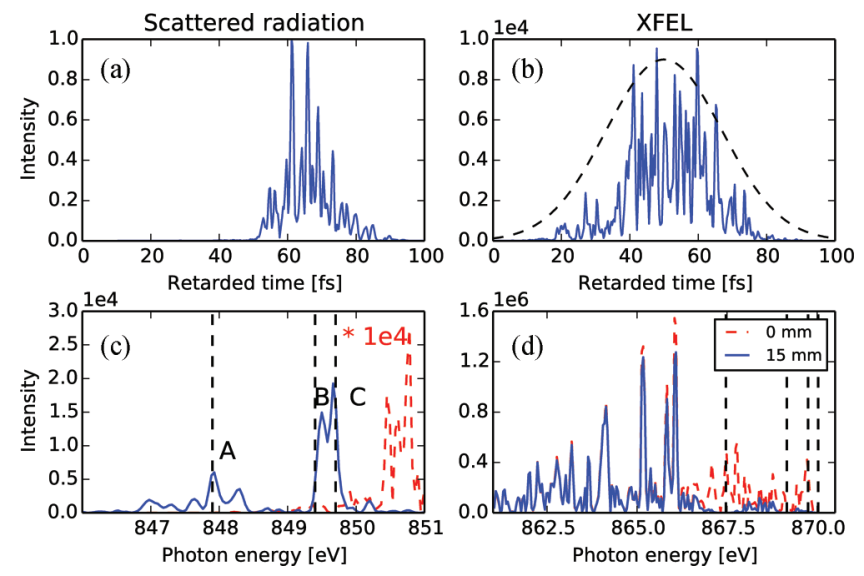

FIG. 3. (Color online) Single-shot temporal profile and spectrum for the scattered radiation [panels (a) and (c)] and XFEL [panels (b) and (d)]. Dotted curve in panel (b) depicts the temporal pulse envelope for the ensemble average. Dotted lines in panel (c) show the detuned transitions $\omega_{1 s^{-1} 3 p \rightarrow 2 p^{-1} 3 p}-\Omega$ with a detuning of $1.4 \mathrm{eV}$ and the transitions $\omega_{1 s^{-1} 3 p \rightarrow 2 p^{-1} 3 p}$ and $\omega_{1 s^{-1}} 5 p \rightarrow 2 p^{-1} 5 p$, while A, B, and C mark the three distinct emission peaks. The dotted line in panel (d) shows the excitation resonances $1 s^{-1} 3 p$ to $1 s^{-1} 6 p$.

incoming and emitted radiation can be seen in Fig. 3. The spectrum of the XFEL radiation at the entrance and end of the gas cell for a typical SASE pulse can bee seen in Fig. 3(d). The transmitted XFEL pulse at the end of the medium shows strong absorption around the resonances. The Raman scattered radiation is depicted in Fig. 3(c). The spectrum shows three distinct peaks $\mathrm{A}, \mathrm{B}$, and $\mathrm{C}$ with some additional broadband background.

To understand the structure of the spectrum it is useful to look at an effective excitation cross section for the specific incoming SASE pulse as a function of the propagation distance. The effective cross section is defined as the product of the XFEL spectrum at position $z$ with the Lorentzian absorption profiles of the excitation resonances weighted with the dipole moment of the respective transition and can be seen in Fig. 4(a). With the help of this plot it is possible to determine the leading spectral spikes and their detuning from the closest resonance governing the scattering process. Figure 4 shows that in the beginning of the medium two strong spikes close to the $1 s-3 p$ resonance and the $1 s-5 p$ resonance dominate the scattering process. These contributions result in the double-peak structure B, C of the emission spectrum in Fig. 3(c). Both spikes are close to the resonance with a detuning of 20 and $28 \mathrm{meV}$, respectively. The peaks B and $\mathrm{C}$ are shifted by the same detuning from the emission transition [dashed lines in Fig. 3(c)]. With propagation these two driving spectral spikes get absorbed and a third spike takes over the amplification. Figure 4(b) shows the detuning $\Omega$ of the two strongest amplifying spikes as a function of propagation distance $z$. In this case the spike at $866 \mathrm{eV}$ on the very left of Fig. 4(a) is the dominating spectral spike from $10-\mathrm{mm}$ propagation distance on. This spike is $1.4 \mathrm{eV}$ detuned to the left of the $1 s-3 p$ resonance. This results in the third speak $\mathrm{A}$ at $848 \mathrm{eV}$ in Fig. 3(c) with a detuning of $1.4 \mathrm{eV}$ from the emission resonance. This shows that propagation can lead to a rich structure in the scattered radiation, since 

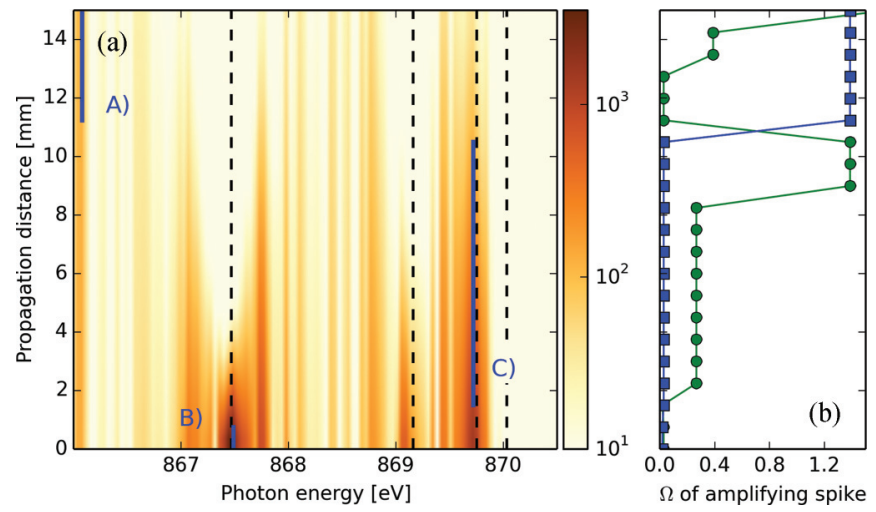

FIG. 4. (Color online) (a) Excitation cross section for a single SASE shot shown as a function of propagation distance. The excitation cross section is the spectrum multiplied with the absorption profiles of resonances weighted with the transition dipole. Black dotted lines mark resonances while the blue line shows the position of the main amplifying spectral spike (b) Detuning $\Omega$ of the strongest (blue squares) and second strongest (green circles) amplifying spike as a function of propagation distance.

multiple spikes with different individual detunings contribute to the scattering. The Raman scattered pulse is not transform limited and typically consists of five to ten spectral modes (number of driving SASE spikes). Even though each spectral spike from the XFEL pulse itself can be considered coherent, different spikes have random phases with respect to each other. Since the scattering process is driven by multiple spikes with different phases the resulting pulses are not transform limited.

To emphasize the difference in emission line shape between stimulated Raman scattering and stimulated fluorescence, Fig. 5(a) shows the ensemble-averaged emission spectra as a function of the incoming central XFEL photon energy across the $K$ edge. We assumed $2 \times 10^{12}$ incoming photons with $40-\mathrm{fs}$ pulse duration. The stimulated scattering for photon energies below $870.2 \mathrm{eV}$ results in a much broader emission structure

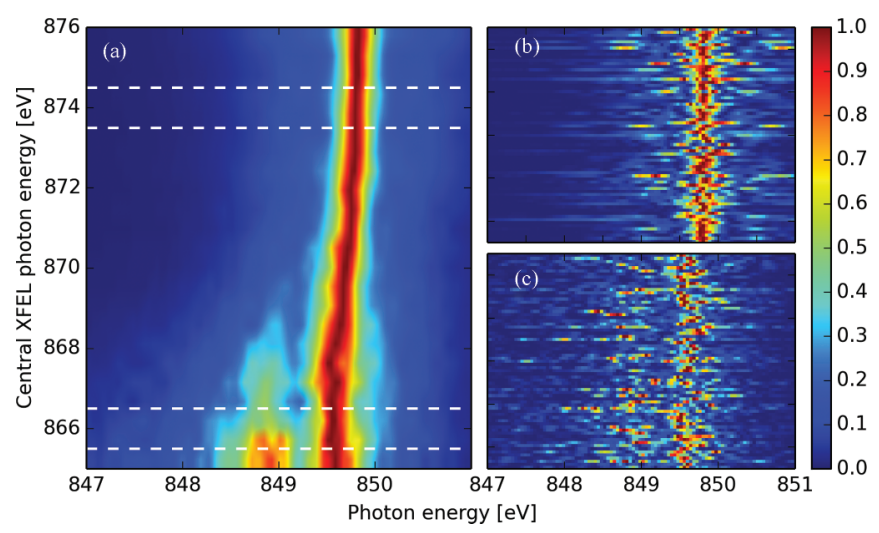

FIG. 5. (Color online) (a) Ensemble-averaged outgoing spectra for different central XFEL photon energies. The ensemble average for each central XFEL photon energy is calculated from 120 single shots. Each row is normalized to its maximum value. Panel (b) shows normalized single-shot emission spectra with central XFEL photon energies between the white dotted lines at 873.5 and $874.5 \mathrm{eV}$, while panel (c) shows single shots between 865.5 and $866.5 \mathrm{eV}$ central XFEL photon energy.

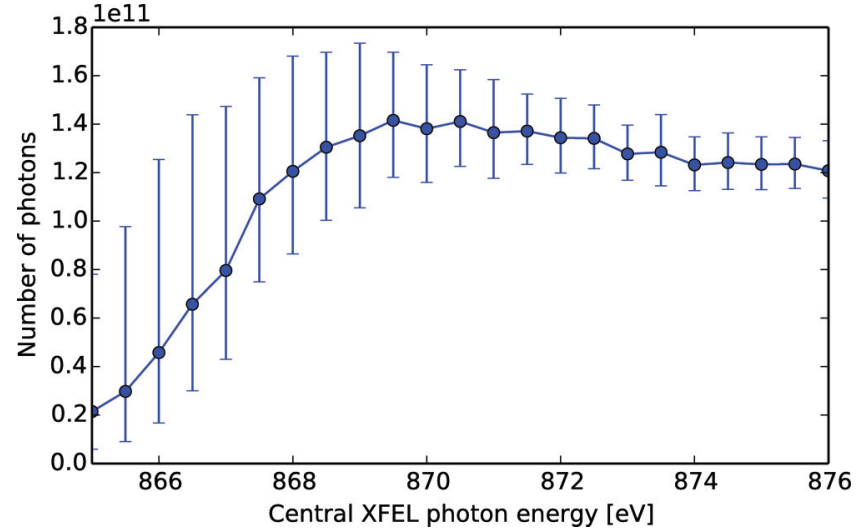

FIG. 6. (Color online) Number of photons in the Raman scattered pulse of a function of central XFEL photon energy. The error bars show the standard deviation for the ensemble of 120 shots for each point.

with a width of $2 \mathrm{eV}$ compared to stimulated fluorescence for photon energies above the $K$ edge. Due to reabsorption of photons around the transition at $849.3 \mathrm{eV}$ it looks like two emission peaks appear for XFEL photon energies below 868 eV. Single-shot-stimulated scattering spectra in Fig. 5(c) reveal the great variation of the emission line shape with a stochastic shift of the maximum position from shot to shot. The individual spikes in the emission have a linewidth of $0.1 \mathrm{eV}$, corresponding to the spectral coherence of the SASE ensemble. For single-shot emission spectra above the $K$ edge [Fig. 5(b)] the line shape is stable and reproducible from shot to shot. Some shots show a broader line shape which is due to saturation broadening.

Despite the overall broad bandwidth of the XFEL pulses, the central XFEL photon energy has a big impact on the Raman gain, i.e., the number of photons in the line emission. Figure 6 shows the number of emitted photons around $849 \mathrm{eV}$ as a function of the incoming central XFEL photon energy, assuming $2 \times 10^{12}$ photons in the incoming XFEL pulses. The Raman gain increases as a function of the central photon energy and reaches a maximum at around the $K$ edge at $870.2 \mathrm{eV}$. Pumping around the $K$ edge there is a significant contribution from stimulated scattering as well as amplified $K \alpha$ fluorescence, leading to a maximum in the emitted number of photons. Beyond the $K$ edge the gain decreases again, reflecting the decrease of the contribution due to resonant scattering. It should also be noted that the variance in the photon emission is larger in the case of resonance scattering. The Raman gain shows large shot-to-shot fluctuations, since the variation of the intensity distribution of the SASE spikes within a resonance window is basically $100 \%$. This large fluctuation in signal gain was also observed in the experiment. This observation is in contrast to Ref. [28], where it was argued that the Raman gain is independent of the exciting laser bandwidth. In that approach, the back reaction on the incoming pump field was neglected and the study considered only a weak pump-field interaction, leaving most atoms in the ground state. In Refs. [37,38] it was shown that propagation effects like dispersion, group velocity, and absorption indeed make the Raman gain depend on the laser bandwidth, in agreement with our observation. 


\section{HIGH RESOLUTION BY COVARIANCE ANALYSIS}

Considering the multispiked, stochastic spectra of stimulated Raman scattering with a SASE XFEL pulse, the outlook on high-resolution X-ray spectroscopy with these sources looks rather grim. In the following we, however, demonstrate that, with the help of statistical analysis of a large ensemble of recorded single spectra with stochastic XFEL pulses, it is possible to obtain high spectral resolution of the scattering process, in a statistical sense. In traditional resonant X-ray Raman scattering spectroscopy narrowband radiation is scanned across the resonances to achieve a high spectral resolution [39]. The resolution in this case is limited by the bandwidth of the incoming radiation and the detector. In the case of SASE pulses with a bandwidth of typically several electron volts, i.e., much larger than the typical width of core-excited resonances in the soft x-ray domain, scanning the resonances is not possible. The structured spectrum of the SASE pulses, consisting of coherent spikes of an average width small compared to the resonance widths, will, however, result in high-energy resolution in a statistical sense. Since each spectrally narrow, coherent spike can drive the scattering process, the scanning with a narrowband source is replaced by a statistical analysis over an ensemble of shots. The spiky structure of the SASE pulse is actually exploited in this case and the spectral resolution is limited by the average width of a spectral spike or, in other words, the spectral coherence of the SASE source.

By doing a covariance analysis [40,41] over an ensemble of single-pulse spectra, the analog of a high-resolution experiment with coherent narrowband pulses can be recovered. We demonstrate this new method based on an ensemble of simulated spectra. The two-dimensional covariance of the ensemble of transmitted, outgoing spectra is defined as

$$
\operatorname{Cov}\left(\omega_{1}, \omega_{2}\right)=\left\langle I\left(\omega_{1}\right) I\left(\omega_{2}\right)\right\rangle-\left\langle I\left(\omega_{1}\right)\right\rangle\left\langle I\left(\omega_{2}\right)\right\rangle .
$$

In Fig. 7 we show the covariance map for an ensemble of 6000 simulated spectra, zoomed in the region of $848<\omega_{2}<$ $851 \mathrm{eV}$ and $866.5<\omega_{2}<870 \mathrm{eV}$. We assume incoming XFEL pulses of $867 \mathrm{eV}$ central photon energy and an average pulse duration of $40 \mathrm{fs}$, which corresponds to a spectral coherence of $0.1 \mathrm{eV}$. The map can be read similar to a high-resolution RIXS is already defined and explained in line 26 and following map, i.e., $\omega_{1}$ can be interpreted as the incoming photon energy of a scattering experiment with a coherent narrowband source, and $\omega_{2}$ can be interpreted as the emitted photon energy. The plot clearly shows the anomalous linear dispersion of the resonant scattering process. A clear separation between scattering process via the $3 p, 4 p$, and $5 p$ core-excited states can be discerned. Moreover, for the $1 s-3 p$ resonance it is possible to see two separate emission lines, corresponding to the final state doublet $2 p^{-1} 3 p^{1} S_{0}$ and $2 p^{-1} 3 p^{1} D_{2}$. For the higher-lying resonances the energy spacing of the final state doublet is too small compared to the spectral coherence of $0.1 \mathrm{eV}$ of the incoming XFEL pulse, resulting in only a single emission line (see Table I). Due to the central XFEL photon energy of $867 \mathrm{eV}$ and smaller excitation cross section to the $4 p, 5 p$, and $6 p$ levels, the emission lines for those higher-lying excitation resonances are less pronounced. The width of the scattering features in

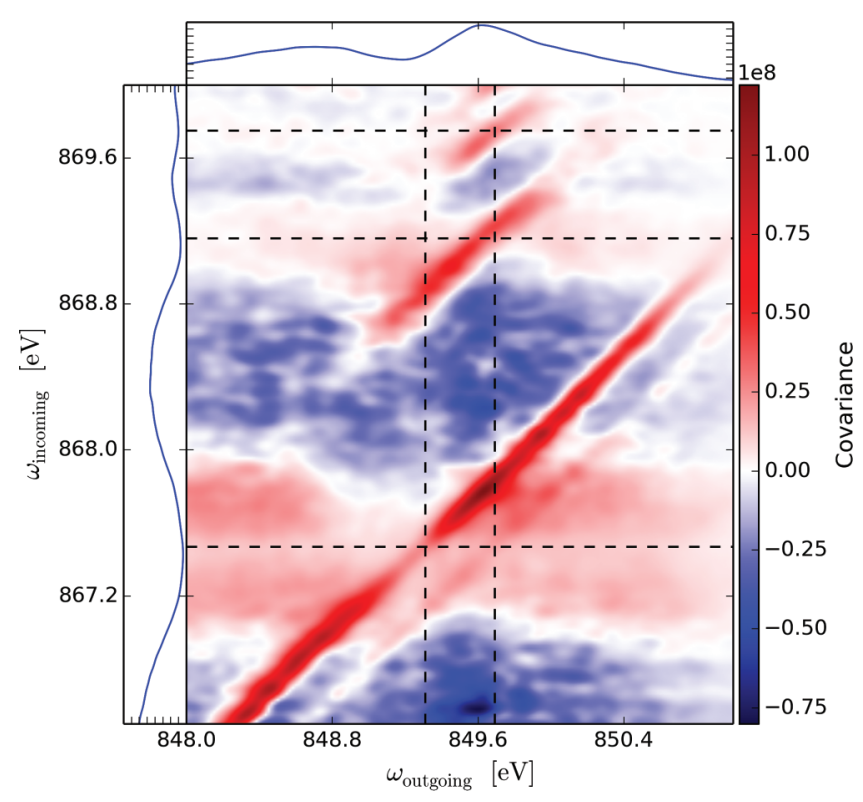

FIG. 7. (Color online) Covariance map for an ensemble of 6000 simulated spectra after $10 \mathrm{~mm}$ of propagation. The horizontal dashed lines mark the excitation resonances. The insets on both axes show the ensemble-averaged spectral intensity.

the covariance plot are $\approx 0.1 \mathrm{eV}$, i.e., corresponding to the incoming spectral coherence of the SASE XFEL pulses.

The resolution of resonant scattering with a stochastic, incoherent source is hence determined by the spectral coherence (average spectral spike width), rather than the overall bandwidth of the incoming pulse. This is similar to observations that the temporal resolution of a nonlinear optical process with chaotic light is determined by the temporal coherence time, rather than the pulse duration, if the temporal, longitudinal, coherence time is smaller than typical relaxation processes or typical time constant in the system [42-44]. Increasing the pulse duration of the SASE pulses, the spectral coherence can be further decreased, resulting in a narrowing of the emission lines and an increased spectral resolution.

\section{CONCLUSION}

In this paper we presented a one-dimensional model to study the propagation of intense, incoherent $\mathrm{x}$-ray pulses through a resonant gas medium. Resonantly exciting neon below the $K$ edge with broadband SASE pulses leads to stimulated electronic x-ray Raman scattering in the forward direction. The inelastic scattering process is seeded by the spectral tails of the XFEL pulse. For typical XFEL parameters achievable at currently operating XFEL sources our model predicts up to $10^{11}$ photons in the Raman scattered pulse, along with a high XFEL transmission of around 0.5 . The Raman scattered pulses show a rich structure of emission lines due to pumping with incoherent XFEL pulses, characterized by several mutually incoherent intensity spikes of small bandwidth. We also demonstrated that, with the help of statistical analysis, a high spectral resolution of the scattering process can be obtained, despite pumping with incoherent radiation. Using the method of covariance mapping it is 
possible to differentiate between the several resonant scattering channels in neon. The resolution is thereby limited by the spectral coherence of the XFEL radiation and not by the overall broad spectral bandwidth. Extending this method to more complex systems and molecules might make it possible to study vibrational dynamics with XFEL radiation.
[1] L. J. P. Ament et al., Rev. Mod. Phys. 83, 705 (2011).

[2] S. Mukamel, Principles of Nonlinear Optical Spectroscopy (Oxford University Press, New York, Oxford, 1999).

[3] V. Ayvazyan et al., Eur. Phys. J. D 37, 297 (2006).

[4] P. Emma et al., Nat. Photonics 4, 641 (2010).

[5] T. Ishikawa et al., Nat. Photonics 6, 540 (2012).

[6] E. Allaria et al., Nat. Photonics 6, 699 (2012).

[7] W. Ackermann et al., Nat. Photonics 1, 336 (2007).

[8] S. Tanaka and S. Mukamel, Phys. Rev. Lett. 89, 043001 (2002).

[9] M. Oura, Plasma Sci. Technol. 12, 353 (2010).

[10] S. Mukamel, D. Healion, Y. Zhang, and J. D. Biggs, Ann. Rev. Phys. Chem. 64, 101 (2013).

[11] C. Weninger et al., Phys. Rev. Lett. (to be published).

[12] F. Gel'mukhanov and H. Ågren, Phys. Rep. 312, 87 (1999).

[13] E. Hudis, P. L. Shkolnikov, and A. E. Kaplan, J. Opt. Soc. Am. B 11, 1158 (1994).

[14] E. E. Fill, S. J. van Enk, J. Zhang, and P. Lambropoulos, Phys. Rev. A 54, 5374 (1996).

[15] H. Ren, J. D. Biggs, and S. Mukamel, J. Raman Spectrosc. 44, 544 (2013).

[16] R. Bonifacio, C. Pellegrini, and L. Narducci, Opt. Commun. 50, 373 (1984).

[17] A. Kondratenko and E. Saldin, Part. Accel. 10, 207 (1980).

[18] S. Krinsky and R. L. Gluckstern, Phys. Rev. Spec. Top.-Accel. Beams 6, 050701 (2003).

[19] E. L. Saldin, E. A. Schneidmiller, and M. V. Yurkov, Opt. Commun. 148, 383 (1998).

[20] D. V. Morgan, M. Sagurton, and R. J. Bartlett, Phys. Rev. A 55, 1113 (1997).

[21] N. Rohringer et al., Nature (London) 481, 488 (2012).

[22] N. Rohringer and R. London, Phys. Rev. A 80, 013809 (2009).

[23] Y.-P. Sun, J.-C. Liu, C.-K. Wang, and F. Gel'mukhanov, Phys. Rev. A 81, 013812 (2010).
[24] O. Larroche, D. Ros, A. Klisnick, A. Sureau, C. Möller, and H. Guennou, Phys. Rev. A 62, 043815 (2000).

[25] C. M. Kim, K. A. Janulewicz, and J. Lee, Phys. Rev. A 84, 013834 (2011).

[26] R. D. Cowan, The Theory of Atomic Structure and Spectra (University of California Press, Berkeley, 1981).

[27] B. Fidel, E. Heyman, R. Kastner, and R. Ziolkowski, J. Comput. Phys. 138, 480 (1997).

[28] M. G. Raymer and J. Mostowski, Phys. Rev. A 24, 1980 (1981).

[29] P. Meystre and M. Sargent, Elements of Quantum Optics (Springer, New York, 1999).

[30] N. Rohringer and R. Santra, Phys. Rev. A 86, 043434 (2012).

[31] B. Bidégaray, A. Bourgeade, and D. Reignier, J. Comput. Phys. 170, 603 (2001).

[32] A. Singer et al., Phys. Rev. Lett. 111, 034802 (2013).

[33] G. Vannucci and M. C. Teich, Appl. Opt. 19, 548 (1980).

[34] N. Rohringer and R. Santra, Phys. Rev. A 76, 033416 (2007).

[35] V. Kimberg and N. Rohringer, Phys. Rev. Lett. 110, 043901 (2013).

[36] P. Eisenberger, P. M. Platzman, and H. Winick, Phys. Rev. Lett. 36, 623 (1976).

[37] R. L. Carman, F. Shimizu, C. S. Wang, and N. Bloembergen, Phys. Rev. A 2, 60 (1970).

[38] A. M. Zheltikov, Phys. Rev. A 76, 033825 (2007).

[39] S. Carniato et al., J. Electron Spectrosc. Relat. Phenom. 181, 116 (2010).

[40] L. J. Frasinski, K. Codling, and P. A. Hatherly, Science 246, 1029 (1989).

[41] L. J. Frasinski et al., Phys. Rev. Lett. (to be published).

[42] Y. Oseledchik and A. I. Burshtein, Radiofizika 26, 698 (1983).

[43] N. Morita and T. Yajima, Phys. Rev. A 30, 2525 (1984).

[44] K. Meyer, C. Ott, P. Raith, A. Kaldun, Y. Jiang, A. Senftleben, M. Kurka, R. Moshammer, J. Ullrich, and T. Pfeifer, Phys. Rev. Lett. 108, 098302 (2012). 